\title{
Technical note: Rapid mineral determination in forages by X-ray fluorescence
}

\author{
P. Berzaghi, ${ }^{* 1}$ A. Lotto, $†$ M. Mancinelli, $\ddagger$ and F. Benozzo* \\ *Department of Animal Production and Health, University of Padua, 35020 Legnaro (PD), Italy \\ †Nutristar S.p.A., 42122 Reggio Emilia (RE), Italy \\ fLaboratorio Analisi Zootecniche, 46023 Gonzaga (MN), Italy
}

\section{ABSTRACT}

A large portion of the cow's ration is composed of forages that can vary greatly in mineral concentrations, which may affect animal performance and health. Current methods for mineral analysis require sample destruction either through wet or dry ashing and complex analytical techniques for individual minerals. Energy dispersive X-ray fluorescence (EDXRF) is a nondestructive, multi-mineral, spectroscopy technique, which makes mineral quantification simple, direct, and affordable. The study objective was to evaluate the prediction performance of EDXRF of $\mathrm{Na}, \mathrm{Mg}, \mathrm{P}$, $\mathrm{S}, \mathrm{Cl}, \mathrm{Ca}, \mathrm{K}, \mathrm{Mn}, \mathrm{Fe}, \mathrm{Cu}$, and $\mathrm{Zn}$ concentrations in forages. Twelve certified plant samples with a wide range in mineral concentrations were used to develop calibrations, and 35 forages (18 alfalfa hays, 10 grass hays, 7 corn silages) with measured mineral concentrations, which were collected over several years from 3 proficiency programs, were used as an independent validation set. All the samples were previously dried and finely ground and were prepared by compressing them into a round dense pellet, $40 \mathrm{~mm}$ in diameter, using a 40-ton pneumatic laboratory press. Samples were scanned using an EDXRF instrument enhanced for lighter minerals like $\mathrm{Na}$ and $\mathrm{Mg}$. Samples were scanned at $20 \mathrm{keV}$ and at $40 \mathrm{KeV}$ associated with an $\mathrm{Al}$ filter, for a total analysis time of approximately $6 \mathrm{~min}$. Calibrations were developed with Bruker SpectraEDX (Bruker, Hamburg, Germany) software and optimized to minimize the standard error of calibration. All of the minerals had acceptable calibration performance with coefficient of determination ranging from $0.93(\mathrm{P})$ and $0.99(\mathrm{Cl}, \mathrm{Ca}$, and $\mathrm{Mn})$ and coefficients of variation within 5 to $14 \%$, which are similar to the coefficients of variation of the reference analysis. The coefficients of variation for $\mathrm{Na}$ was an exception, with a coefficients of variation of $29 \%$. The validation set obtained similar statistical results as that observed in calibration. The

\footnotetext{
Received March 13, 2018.

Accepted July 27, 2018.

${ }^{1}$ Corresponding author: paolo.berzaghi@unipd.it
}

root mean square error of prediction corrected for bias was similar to the standard error of calibration, indicating that it is possible to build a robust calibrations that performed well across different type of forages by using 12 reference samples with a sufficient range in mineral concentrations that were determined accurately. A bias correction was necessary to improve prediction accuracy only for $\mathrm{K}(-0.23 \%$ dry matter $)$ and $\mathrm{Ca}(-0.16 \%$ dry matter). Energy dispersive X-ray fluorescence demonstrated the ability to be an accurate, direct, and simple technique for forage mineral analysis.

Key words: mineral, forage, X-ray fluorescence

\section{Technical Note}

Feeding a balanced ration for all nutrients, including minerals, is crucial for maintaining high levels of milk production and animal health (Goff, 2006). Forages usually represent a large portion of the ration, but also have a large variability in nutritional quality (Dairy One, 2018) that should be monitored regularly (St-Pierre and Cobanov, 2007). The current and most used analytical techniques such as atomic absorption and inductively coupled plasma mass spectrometry require extensive sample preparation, like acid hydrolysis (Tagami, 2006). This sample preparation, besides being time consuming and producing reagents waste, is not suitable for $\mathrm{Cl}$ because a significant portion of this halogen mineral is lost during the acid hydrolysis (Tagami, 2006). In comparison, energy dispersive Xray fluorescence (EDXRF) is a nondestructive spectroscopy technique, which requires minimum sample preparation, that include drying, grinding, and pressing into a pellet. The EDXRF can measure many minerals simultaneously, which may have large ranges in mineral concentrations, making it attractive for plant and forage analysis (Djingova, 2013). The study objective was to evaluate the accuracy of EDXRF as a method to determine the concentration of macro- and some microminerals in forage samples.

The study was performed using an energy dispersive X-ray spectrometer (S2 Ranger LE, Bruker, Hamburg, Germany) with a Pd anode X-ray tube, Peltier cooled 
Table 1. List of certified reference standards used for calibration

\begin{tabular}{ll}
\hline Type of material & Reference code \\
\hline Fescue plant & IPE965 \\
Corn plant & IPE723 \\
Wheat straw & IPE962 \\
Wheat straw & IPE881 \\
Alfalfa plant & IPE966 \\
Grass mixture & IPE952 \\
Corn stalk & IPE968 \\
Mixed Polish herbs & INCT-MPH-2 \\
Pinus taeda leaves & 1575A \\
Fagus sylvatica leaves & BCR100 \\
Olive leaves & BCR62 \\
Hay powder & BCR129 \\
\hline
\end{tabular}

detector, and maximum power of $50 \mathrm{~W}, 2 \mathrm{~mA}$, or 50 $\mathrm{KeV}$. About $5 \mathrm{~g}$ of finely ground sample was pressed into a pellet of $40 \mathrm{~mm}$ in diameter using a 40-ton pneumatic laboratory press (International Crystal Laboratories, Garfield, NJ). The pellet was placed within a round holder, inserted in a shielded chamber that was evacuated before it was irradiated with X-ray at different energy levels to excite different minerals until 100,000 cps was reached. First, samples were irradiated at 20 $\mathrm{KeV}$ without any filter for the determination of lighter minerals ( $\mathrm{Na}, \mathrm{Mg}, \mathrm{P}, \mathrm{S}, \mathrm{Cl}$ ) and then at $40 \mathrm{KeV}$ with an $\mathrm{Al}$ filter to optimize fluorescence for the heavier $(\mathrm{K}$, $\mathrm{Ca}, \mathrm{Fe}, \mathrm{Mn}, \mathrm{Cu}, \mathrm{Zn}$ ) minerals. The total scan time per sample was approximately $6 \mathrm{~min}$. Calibrations for the different minerals were developed using a set of 12 certified plant samples (Table 1) selected either from the one available from the European Commission Joint Research Center (JRC, Geel, Belgium), and the Wageningen Evaluating Programmes for Analytical Laboratories (WEPAL, Wageningen, the Netherlands) catalogs. Calibrations were developed using Bruker SpectraEDX software by regressing the fluorescence emission at either 20 or $40 \mathrm{KeV}$ scan against the known minerals concentration of the 12 certified standards. Regressions were optimized by selecting either the peak height or peak area to minimize the standard error of regression also known as standard error of calibration (SEC). Validation was performed by comparing EDXRF mineral predictions to the reference mineral analysis of 35 forage samples (18 alfalfa hays, 10 grass hays, and 7 corn silages). Validation samples and their mineral analyses were obtained over years from 3 interlaboratory proficiency programs that provided reference mineral analysis to evaluate laboratory accuracy. Twenty samples were from the National Forage Testing Association (United States), 7 from the University of Piacenza (Italy), and 8 from Bipea (France). Not all the minerals predicted by EDXRF were available for the validation samples, such that $\mathrm{Na}, \mathrm{Mn}$, and Fe were available only for 7 samples and $\mathrm{Cl}$ was not reported for any of the samples from these proficiency programs (Table 2). All of the other minerals had at least 15 validation samples. Because of the lack of validation samples for $\mathrm{Cl}$, 6 forage (2 alfalfa hays, 2 grass hays, 2 corn silages) samples were analyzed for $\mathrm{Cl}$ using the Volhard method (Eur-Lex, 2009).

Calibration performance was evaluated based on the coefficient of determination $\left(\mathbf{R}^{2}\right)$, SEC, and the coefficient of variation of the calibration error $(\mathbf{C V c})$, calculated as SEC $\times 100 /$ mean concentration. Validation statistics were computed by using regression in Microsoft Excel (Microsoft Corp., Redmond, WA) to compare reference values of validation samples and EDXRF predicted values and determining the $\mathrm{R}^{2}$, the bias (difference between average reference values minus average EDXRF predictions), also known as systematic error, the prediction error expressed as root mean square error of prediction (RMSEP), and the standard error of prediction (SEP), which is the RMSEP after correction of the systematic error (Dardenne, 2009).

Table 2. Evaluation of energy dispersive X-ray fluorescence prediction compared with validation standards

\begin{tabular}{|c|c|c|c|c|c|c|c|c|c|}
\hline $\begin{array}{l}\text { Mineral } \\
\text { (DM basis) }\end{array}$ & \multicolumn{9}{|c|}{ Calibration statistics $^{1}$} \\
\hline $\mathrm{Na}, \mathrm{mg} / \mathrm{kg}$ & 7 & 929 & 1,053 & 200 & 3,300 & 0.984 & 153 & -45 & 158 \\
\hline P, \% & 35 & 0.27 & 0.07 & 0.05 & 0.39 & 0.957 & 0.02 & 0.00 & 0.02 \\
\hline S, \% & 20 & 0.21 & 0.09 & 0.08 & 0.38 & 0.985 & 0.02 & -0.02 & 0.01 \\
\hline $\mathrm{Cl}, \%$ & 6 & 0.35 & 0.04 & 0.05 & 0.70 & 0.987 & 0.05 & -0.04 & 0.03 \\
\hline $\mathrm{Fe}, \mathrm{mg} / \mathrm{kg}$ & 7 & 506 & 406 & 192 & 1,361 & 0.995 & 160 & -126 & 107 \\
\hline $\mathrm{Cu}, \mathrm{mg} / \mathrm{kg}$ & 15 & 8 & 4 & 2 & 18 & 0.809 & 3 & -2 & 2 \\
\hline $\mathrm{Zn}, \mathrm{mg} / \mathrm{kg}$ & 35 & 40 & 60 & 9 & 360 & 0.996 & 7 & -4 & 6 \\
\hline
\end{tabular}

${ }^{1} \mathrm{n}=$ number of validation standards; RMSEP = root mean squared error of prediction; bias = average difference between predicted and reference values; $\mathrm{SEP}=$ root mean squared error of prediction corrected for bias. 
Table 3. Calibration performance for energy dispersive X-ray fluorescence using certified reference standards

\begin{tabular}{lcccccccc}
\hline & \multicolumn{7}{c}{ Calibration statistics $^{1}$} \\
\cline { 2 - 9 } $\begin{array}{l}\text { Mineral } \\
\text { DM basis) }\end{array}$ & $\mathrm{n}$ & Mean & $\mathrm{SD}$ & $\mathrm{CVc}$ & Minimum & Maximum & $\mathrm{R}^{2}$ & RMSEC \\
\hline $\mathrm{Na}, \mathrm{mg} / \mathrm{kg}$ & 6 & 340 & 488 & 29 & 10 & 1,300 & 0.95 & 100 \\
$\mathrm{Mg}, \%$ & 11 & 0.23 & 0.21 & 13 & 0.05 & 0.65 & 0.97 & 0.03 \\
$\mathrm{P}, \%$ & 10 & 0.27 & 0.12 & 11 & 0.05 & 0.43 & 0.93 & 0.03 \\
$\mathrm{~S}, \%$ & 11 & 0.23 & 0.12 & 4 & 0.08 & 0.43 & 0.98 & 0.01 \\
$\mathrm{Cl}, \%$ & 12 & 0.64 & 0.61 & 6 & 0.02 & 2.01 & 0.99 & 0.04 \\
$\mathrm{~K}, \%$ & 11 & 2.44 & 1.56 & 11 & 0.44 & 5.18 & 0.97 & 0.27 \\
$\mathrm{Ca}, \%$ & 11 & 0.98 & 1.28 & 10 & 0.04 & 4.46 & 0.99 & 0.10 \\
$\mathrm{Mn}, \mathrm{mg} / \mathrm{kg}$ & 12 & 201 & 330 & 5 & 7 & 1,162 & 0.99 & 10 \\
$\mathrm{Fe}, \mathrm{mg} / \mathrm{kg}$ & 10 & 194 & 178 & 14 & 46 & 538 & 0.97 & 27 \\
$\mathrm{Cu}, \mathrm{mg} / \mathrm{kg}$ & 12 & 6 & 4 & 33 & 2 & 15 & 0.97 & 2 \\
$\mathrm{Zn}, \mathrm{mg} / \mathrm{kg}$ & 11 & 36 & 20 & 8 & 8 & 82 & 0.98 & 3 \\
\hline
\end{tabular}

${ }^{1} \mathrm{n}=$ number of calibration standards; RMSEC $=$ root mean squared error of calibration; $\mathrm{CVc}=$ coefficient of variation in calibration $(\mathrm{RMSEC} \times 100 /$ mean $)$.

Calibration statistics (Table 3 ) indicated $\mathrm{R}^{2}$ greater than 0.93 for all minerals. Because lighter minerals $(\mathrm{Na}$, $\mathrm{Mg}$, and $\mathrm{P}$ ) have lower X-ray fluorescence, their slightly lower $\mathrm{R}^{2}$ compared with the other minerals was not unexpected, as they are more difficult to quantify by EDXRF. Nonetheless, even Na, the lightest mineral, demonstrated good calibration statistics, likely because the instrument was an enhanced version for detecting lighter minerals. The largest SEC was $\mathrm{K}$ at $0.27 \% \mathrm{DM}$, which was expected because it had the largest range in mineral concentration. Calcium, which is important in a balanced dairy ration (NRC, 2001), had an $\mathrm{R}^{2}$ of 0.99 and SEC of $0.10 \%$ DM, indicating accurate quantification. The heavier minerals such $\mathrm{Mn}, \mathrm{Fe}, \mathrm{Cu}$, and $\mathrm{Zn}$ demonstrated excellent performances with SEC for the heaviest $\mathrm{Cu}$ and $\mathrm{Zn}$ close to the lower limit of detection of 1 to $3 \mathrm{mg} / \mathrm{kg}$ (Ivanova, 1999).

Evaluating the relative errors as indicated by $\mathrm{CVc}$, the best calibrations were obtained for $\mathrm{S}, \mathrm{Cl}, \mathrm{Mn}$, and Zn with a CVc of $<10 \%$ (Table 3 ). Then a second group of minerals ( $\mathrm{Mg}, \mathrm{P}, \mathrm{K}, \mathrm{Ca}$, and $\mathrm{Fe}$ ) yielded $\mathrm{CVc}$ between 10 and $14 \%$. The poorest mineral calibrations were $\mathrm{Na}$ and $\mathrm{Cu}$. Sodium had a CVc of $29 \%$, which can be explained by the limitations of EDXRF detecting the lighter minerals (Jenkins, 1999), whereas Cu performance is related to the low concentration (less than $10 \mathrm{mg} / \mathrm{kg}$ ), which is close to the EDXRF lower limit of detection. A SEC of $2 \mathrm{mg} / \mathrm{kg}$ is about the lowest possible error for $\mathrm{Cu}$ using this analytical technique. The coefficients of variation of reference analyses for the certified materials typically varied between 5 and $15 \%$, which is similar to the range of $\mathrm{CVc}$ for most minerals. However, $\mathrm{Cu}$, having a low average concentration, had a coefficient of variation analysis of $25 \%$, not far from calibration results. Although the $\mathrm{CVc}$ for the different minerals may be large, the calibration performances were comparable to the precision of analysis reported in the certificates of the certified materials. The only exception is $\mathrm{Na}$ that had the largest $\mathrm{CVc}$, which was greater than reported in the reference standards.

Even with some limitation with reference analysis for some minerals $(\mathrm{Na}, \mathrm{Cl}, \mathrm{Mn}$, and $\mathrm{Fe}$ ), the validation data set provided a solid confirmation of the results obtained through calibration. With the only exception of $\mathrm{Cu}\left(\mathrm{R}^{2}=0.81\right)$, all of the other minerals had an $\mathrm{R}^{2}$ of 0.93 or greater. The validation error (RMSEP) for most of the minerals confirmed the SEC with the exception of $\mathrm{Ca}, \mathrm{Fe}$, and $\mathrm{Zn}$. Calcium had a SEC of $0.10 \%$, but in validation the RMSEP was nearly double at $0.21 \%$ even with a $\mathrm{R}^{2}$ of 0.998 . The bias for $\mathrm{Ca}$ was on average $-0.16 \%$, which can happen when comparing results coming from different laboratories and if we apply this correction systematically to all EDXRF Ca results, the corrected prediction error (SEP) would be $0.13 \%$ DM, which is very similar to the calibration performance. Iron had also a larger RMSEP, and even with a bias adjustment, the prediction error remained much larger $(\mathrm{SEP}=107 \mathrm{mg} / \mathrm{kg})$ than what could be expected from the magnitude of the calibration error (SEC $=27 \mathrm{mg}$ / $\mathrm{kg}$ ). The validation data set had a wider range in $\mathrm{Fe}$ concentration than the calibration database, and the 2 validation samples that had $\mathrm{Fe}$ concentration exceeding the upper calibration range had the largest errors. In this case it would be advisable to update the calibration using a wider range in Fe concentration. Similarly, the validation database for $\mathrm{Zn}$ had 2 samples that exceeded the calibration range and the validation sample with the highest concentration $(360 \mathrm{mg} / \mathrm{kg})$ had the largest prediction error. Removing that sample, the RMSEP would decrease to 5 and the SEP to 4 , not too far from the calibration error. Like $\mathrm{Ca}, \mathrm{K}$ had a fairly large bias value $(-0.23 \%)$, which was similar in magnitude to the 
RMSEP (0.26\%). Adjusting the XRF predictions for systematic bias would lower SEP at $0.13 \%$, even better than the calibration results.

Limited literature is available about the prediction performance of XRF for forage, but there are reports of using EDXRF for mineral analysis of plant material. Ivanova (1999) reported reliable prediction for $\mathrm{Ca}$, Fe, K, Mn, and Zn for different plant sources. A few samples of hay and straw along cereals and mixed feeds were analyzed by Pukhovski (2002), with good predicting performances for $\mathrm{P}, \mathrm{K}, \mathrm{Ca}, \mathrm{Mn}, \mathrm{Fe}$, and Zn. The prediction errors observed in this study indicate that using EDXRF to analyze forages would improve the accuracy of minerals for ration formulation compared with using mineral analysis from near-infrared spectroscopy (Clark et al., 1987), as it is often used. From a practical point of view, improving forage mineral analysis would allow more accurate supplementation of only the mineral needed, with the reduction of feeding costs, and of unnecessary excretions, which may affect the environment.

Mineral analysis by EDXRF using modern instruments can provide accurate quantification of the major macro- and some microminerals in forage samples. Simple sample preparation and the lack of using reagents make this technique easy to implement even in small forage laboratories, providing the opportunity to accurately measure forage mineral concentrations using a cost-effective method. Having a sample preparation similar to what is necessary for near infrared analysis (Abrams, 1989), EDXRF could be easily integrated into the operations of forage laboratories that use near infrared technology and enhance their ability to measure minerals.

\section{ACKNOWLEDGMENTS}

Funded by the Italian Ministry of Agriculture and Forestry, Rome, Italy, project MARIPA 2009.

\section{REFERENCES}

Abrams, S. M. 1989. Sample Preparation. Agric. Handbook No. 643. ARS-USDA, Washington, DC.

Clark, D. H., H. F. Mayland, and R. C. Lamnb. 1987. Mineral analysis of forages with near infrared reflectance spectroscopy. Agron. J. 79:485-490.

DairyOne. 2018. Feed composition libraries. Accessed Mar. 8, 2018. http:// dairyone.com/analytical-services/feed-and-forage/feed -composition-library/interactive-feed-composition-library/.

Dardenne, P. 2009. Some considerations about NIR spectroscopy: Closing speech at NIR-2009. 241 NIR News. 21:8-9, 14. https:// doi.org/10.1255/nirn.1165.

Djingova, R. M. 2013. Multielement analytical spectroscopy in plant ionomics research. Appl. Spectrosc. Rev. 485:384-424. https://doi .org/10.1080/05704928.2012.703153.

Eur-Lex. 2009. Laying down the methods of sampling and analysis for the official control of feed. Accessed Mar. 1, 2018. http: //eur-lex.europa.eu/legal-content/EN/TXT/PDF/?uri=CELEX: 32009R0152\&qid $=1519911215030 \&$ from $=$ EN.

Goff, J. P. 2006. Macromineral physiology and application to the feeding of the dairy cow for prevention of milk fever and other periparturient mineral disorders. Anim. Feed Sci. Technol. 126:237-257. https://doi.org/10.1016/j.anifeedsci.2005.08.005.

Ivanova, J. R. D. 1999. Possibilities of ED-XRF with radionuclide sources for analysis of plants. J. Radioanal. Nucl. Chem. 2422:569575. https://doi.org/10.1007/BF02345596.

Jenkins, R. 1999. X-ray fluorescence spectrometry. 2nd ed. John Wiley \& Sons Inc., New York, NY.

NRC. 2001. Nutrient Requirements of Dairy Cattle. 7th rev. ed. Natl. Acad. Press, Washington, DC.

Pukhovski, A. V. 2002. X-ray fluorescence analysis in the Russian State Agrochemical Service: An overview. XRay Spectrom. 31:225-234. https://doi.org/10.1002/xrs.562.

St-Pierre, N. R., and B. Cobanov. 2007. Optimal sampling schedule of diet components: Model robustness to departure from assumptions. J. Dairy Sci. 90:5395-5404.

Tagami, K. S. U. 2006. Determination of chlorine, bromine and iodine in plant samples by inductively coupled plasma-mass spectrometry after leaching with tetramethyl ammonium hydroxide under a mild temperature condition. Anal. Chim. Acta 5701:88-92. https://doi .org/10.1016/j.aca.2006.04.011. 